\title{
Photon radiation with MadDipole
}

\author{
Gehrmann, T ; Greiner, N
}

\begin{abstract}
We present the automation of a subtraction method for photon radiation using the dipole formalism within the MadGraph framework. The subtraction terms are implemented both in dimensional regularization and mass regularization for massless and massive cases and non-collinear-safe observables are accounted for.
\end{abstract}

DOI: https://doi.org/10.1007/JHEP12(2010)050

Posted at the Zurich Open Repository and Archive, University of Zurich

ZORA URL: https://doi.org/10.5167/uzh-41378

Journal Article

Accepted Version

Originally published at:

Gehrmann, T; Greiner, N (2010). Photon radiation with MadDipole. Journal of High Energy Physics, (12):050.

DOI: https://doi.org/10.1007/JHEP12(2010)050 


\title{
Photon Radiation with MadDipole
}

\author{
T. Gehrmann ${ }^{a}$,N. Greiner ${ }^{b}$ \\ ${ }^{a}$ Institut für Theoretische Physik, Universität Zürich, \\ Winterthurerstrasse 190, 8057 Zürich, Switzerland \\ ${ }^{b}$ Department of Physics, University of Illinois at Urbana-Champaign, \\ 1110 West Green Street, Urbana IL, 61801, USA
}

\begin{abstract}
We present the automation of a subtraction method for photon radiation using the dipole formalism within the MadGraph framework. The subtraction terms are implemented both in dimensional regularization and mass regularization for massless and massive cases and non-collinear-safe observables are accounted for.
\end{abstract}




\section{Contents}

1. Introduction 1

2. Dimensional regularization vs. mass regularization 3

3. QED corrections to parton distribution functions 4

4. Non-collinear-safe observables 5

$\begin{array}{lll}4.1 & \text { final-final } & 7\end{array}$

4.2 final-initial 8

4.3 Factorization of the collinear final state singularity 8

5. Implementation and how to use it 9

5.1 Implementation of non-collinear-safe case 11

6. Checks 12

$\begin{array}{lll}\text { 7. Conclusions } & 13\end{array}$

A. Final-final $f \rightarrow f+\gamma$ dipole in dimensional regularization $\quad 14$

$\begin{array}{lll}\text { A.1 } \alpha \text {-cut parameter } & 16\end{array}$

\section{Introduction}

A precise theoretical description of cross sections at particle colliders is required for the reliable extraction of fundamental parameters, for indirect searches for new physics effects, and for a well-founded assessment of background reactions to new physics signatures and their uncertainties. This precision is achieved by inclusion of higher order perturbative corrections from QCD and from the electroweak theory. At hadron colliders, especially the inclusion of next-to-leading order (NLO) QCD appears to be mandatory for many studies. Much effort has been made in the recent past to automate NLO QCD calculations (see $[1,2]$ for reviews).

A priori, NLO electroweak corrections can be expected to be smaller than QCD corrections, owing to the smaller coupling constant. In a variety of situations, this naive argumentation does however not apply. In QCD, quarks and gluons can not be distinguished, and collinear gluon radiation off an outgoing parton can not be identified experimentally. Collinear photon radiation off a lepton leaves a signature in the detector and can thus be identified. As a result, the cancellation of photonic contributions in the virtual and real collinear contributions at NLO is incomplete, and the observable is non-collinear-safe [3]. 
The final state photon selection cuts must be implemented consistently in the theoretical description, both at fixed order and in a parton shower framework [4]. Depending on the treatment of photon radiation in the experimental analysis, the magnitude and shape of the electroweak corrections can differ substantially, due to the occurrence of large logarithmic terms in the photon cuts, as discussed in detail for instance in the electroweak corrections to gauge boson production and decay $[5,6]$. At centre-of-mass energies above the weak gauge boson masses, similar incomplete cancellations occur in the genuine weak corrections, giving rise to large logarithms in the gauge boson masses.

NLO electroweak corrections for hadron collider observables have up to now been derived for gauge boson production [5,6], for gauge boson pair production [7], for Higgs boson production and decay [8-13], for top quark pair production [14,15], single top production [16] and for supersymmetric particle production [17-19]. Much effort has been made recently towards electroweak corrections to final states including jets. For these final states, the experimental treatment of photons observed inside jets [20] can yield noncollinear-safe configurations, resulting in potentially large corrections. A first calculation of this type was accomplished recently with the NLO corrections to $W^{ \pm}+$jet final states [21].

NLO electroweak corrections require two ingredients: the one-loop virtual corrections and the real radiation corrections with one extra final state particle. While the one-loop virtual corrections contain explicit infrared poles from the loop integration, the real radiation contributions develop infrared singularities only when integrated over the real radiation phase space. To handle these real radiation singularities in a process-independent manner, subtraction formalisms have been developed initially in the context of QCD calculations: residue (or FKS) subtraction [22] and variants thereof [23], dipole subtraction [24,25] and antenna subtraction [26-29], all within dimensional regularization. These can in principle be carried over to electroweak calculations. However, some care has to be taken since most electroweak calculations use mass regularization. The dipole subtraction method has been extended to include photonic radiation in [30].

Since NLO electroweak corrections are required for a substantial number of processes, their automation is very much desirable. Driven mainly by QCD calculations, the automation of one-loop corrections for one-loop multi-parton amplitudes has seen enormous progress in the recent past, and first fully automated programs for the calculation of oneloop multi-parton amplitudes are becoming available with the packages CutTools [31,32], BlackHat [33], Rocket [34], GOLEM [35] and Samurai [36], as well as independent libraries [37].

Automation of the real radiation contributions is normally based on a leading order parton-level event generator framework such as MadGraph/MadEvent [38-40], ALPGEN [41], CompHEP/CalcHEP [42]/ [43], SHERPA [44, 45], HELAC [46] or WHIZARD [47]. The generation of dipole terms for subtracting the singular behaviour from the real radiation subprocesses has been automated in various event generators: in the SHERPA framework [48], the TeVJet framework [49], the HELAC framework [50] and in the form of independent libraries [51,52] interfaced to MadGraph. The MadDipole package [53] provides an implementation within MadGraph. An implementation of the residue subtraction method is also available within MadGraph [54]. For a full NLO calculation, the dipole 
terms have to be integrated over the dipole phase space, and added with the virtual corrections to obtain the cancellation of infrared singularities. The HELAC implementation [50] and the MadDipole package [55] provide these integrated dipole terms including all masses and possible phase-space restrictions.

It is the purpose of this work to extend the MadDipole package towards electroweak NLO corrections by automatically generating the unintegrated and integrated dipole subtraction terms for photonic radiation, as defined in [30], and including non-collinear-safe configurations [3].

This paper is structured as follows: in Section 2, we review the differences between mass regularization and dimensional regularization, and their implications for the dipole terms. The treatment of photonic effects in the parton distributions is described in Section 3, and the dipole terms for non-collinear-safe observables are introduced in Section 4. The implementation of photon radiation in MadDipole is documented in Section 5, and checks of it are described in Section 6.

\section{Dimensional regularization vs. mass regularization}

Infrared singularities are an inherent property of quantum field theories. In the dipole formalism this becomes exlicit when integrating over the one particle phase space to obtain the integrated subtraction terms. As the integration over this phase space is divergent one needs a prescription to parametrize the singularities. For QCD calculations dimensional regularization is the most widely used method. The singularities are present in poles of the form $1 / \epsilon^{2}$ and $1 / \epsilon$. Electroweak calculations are mostly done using mass regularization where the actual masses of the fermions and a hypothetical mass parameter of the photon are used as infrared cut-offs. In this case the singularities show up as logarithms of the mass parameter.

In our implementation we offer both possibilities and the user can select which method is used. The implementation within dimensional regularization works in the same way as for the QCD version of MadDipole, described in [53,55].

In handling photon radiation, the main differences are in the initial state radiation collinear singularities, which lack counterparts in the virtual corrections. In QCD these singularities are absorbed by a redefinition of the parton distribution functions. Integrated subtraction terms for processes with initial state partons contain distributions in the momentum fraction $x$ of the parton entering the hard scattering process (for details see [24,25]). A priori the reduced matrix element also depends on $x$, a feature that bears disadvantages for the numerical implementation: the matrix element has to be evaluated at $x \neq 1$ and at $x=1$. In the presence of a parton distribution function this problem can be circumvented by a transformation of variables, thus shifting the dependence of this variable into the parton distribution function. The details and how this is done in MadDipole is described in [55].

The major new aspects in the implementation of photon radiation are the following:

1. Initial state radiation singularities in electroweak processes are regulated by the masses of leptons entering the hard process, resulting in potentially large mass logarithms. In 
contrast to the divergencies absorbed into the parton distribution functions, these mass logarithms have physical significance. Consequently, for electroweak corrections to processes with leptons in the initial state, the user has to choose mass regularization as the appropriate scheme. Furthermore, it is not possible to remove the $x$-dependence from the hard scattering matrix element by a variable redefinition, which thus has to be evaluated up to three times: at $x=1$, and at the values of $x$ corresponding to the initial state radiation off each of the two incoming momenta.

2. For configurations containing only a collinear but not a soft divergence, the summation over the dipole spectator momenta is obsolete, since these configurations can be accounted for by a single dipole term. In the QCD MadDipole implementation, this summation is nevertheless carried out in order to facilitate the colour management. In the electroweak MadDipole implementation, this summation is omitted. For the convenience of the user, all possible spectator terms are generated, and can be invoked manually.

\section{QED corrections to parton distribution functions}

For processes with incoming partons, initial state collinear singularities are absorbed into the parton distribution functions (PDFs). In dimensional regularization this collinear singularity is of the form

$$
\frac{\left(4 \pi \mu^{2}\right)^{\epsilon}}{\Gamma(1-\epsilon)} \frac{1}{\epsilon} P_{j i}(x)
$$

where $P_{j i}$ denotes the Altarelli-Parisi splitting function for the $j \rightarrow i$ splitting.

For example, if one considers gluon radiation off an incoming quark, the absorption of the collinear singularity of 3.1 into the quark PDF leads to

$$
q\left(x, \mu_{F}\right)=q_{0}(x)+\frac{\alpha_{s}}{2 \pi} \int_{x}^{1} \frac{d y}{y} q_{0}(y)\left[\frac{-1}{\Gamma(1-\epsilon)}\left(\frac{4 \pi \mu^{2}}{\mu_{F}^{2}}\right)^{\epsilon} \frac{1}{\epsilon} P_{q q}\left(\frac{x}{y}\right)+C_{q q}\left(\frac{x}{y}\right)\right]
$$

where $\mu_{F}$ is the factorization scale. The value of the coefficient $C_{q q}\left(\frac{x}{y}\right)$ defines the subtraction scheme. The $\overline{M S}$ scheme which is mainly used in QCD just absorbs the singular terms and some trivial constants, so

$$
C_{q q}^{\overline{M S}}(x)=0
$$

For the subtraction of photon radiation, the equivalent expressions for QED corrections using mass regularization are needed. This can be derived by calculating the integrated subtraction terms both in dimensional and mass regularization and comparing the results. A detailed derivation can be found in $[8,56,57]$, so we only quote the results here. According to Eqns. (3.19) and (3.20) of [56], to include inital state radiation at NLO QED of a parton $a$ out of a hadron $h$ with longitudinal momentum fraction $x$, the parton distribution functions 
are modified by

$$
\begin{aligned}
f_{q / \bar{q}}^{(h)}(x) \rightarrow f_{q / \bar{q}}^{(h)}\left(x, \mu_{\mathrm{F}}^{2}\right)- & \frac{\alpha Q_{q}^{2}}{2 \pi} \int_{x}^{1} \frac{\mathrm{d} z}{z} f_{q / \bar{q}}^{(h)}\left(\frac{x}{z}, \mu_{\mathrm{F}}^{2}\right) \\
& \times\left\{\ln \left(\frac{\mu_{\mathrm{F}}^{2}}{m_{q}^{2}}\right)\left[P_{f f}(z)\right]_{+}-\left[P_{f f}(z)(2 \ln (1-z)+1)\right]_{+}+C_{f f}^{\mathrm{DIS}}(z)\right\} \\
- & 3 \frac{\alpha Q_{q}^{2}}{2 \pi} \int_{x}^{1} \frac{\mathrm{d} z}{z} f_{\gamma}^{(h)}\left(\frac{x}{z}, \mu_{\mathrm{F}}^{2}\right)\left\{\ln \left(\frac{\mu_{\mathrm{F}}^{2}}{m_{q}^{2}}\right) P_{f \gamma}(z)+C_{f \gamma}^{\mathrm{DIS}}(z)\right\} \\
f_{\gamma}^{(h)}(x) \rightarrow f_{\gamma}^{(h)}\left(x, \mu_{\mathrm{F}}^{2}\right)- & \frac{\alpha Q_{q}^{2}}{2 \pi} \sum_{a=q, \bar{q}} \int_{x}^{1} \frac{\mathrm{d} z}{z} f_{a}^{(h)}\left(\frac{x}{z}, \mu_{\mathrm{F}}^{2}\right) \\
& \times\left\{\ln \left(\frac{\mu_{\mathrm{F}}^{2}}{m_{q}^{2}}\right) P_{\gamma f}(z)-P_{\gamma f}(z)(2 \ln z+1)+C_{\gamma f}^{\mathrm{DIS}}(z)\right\}
\end{aligned}
$$

with the splitting functions

$$
P_{f f}(z)=\frac{1+z^{2}}{1-z}, \quad P_{f \gamma}(z)=z^{2}+(1-z)^{2}, \quad P_{\gamma f}(z)=\frac{1+(1-z)^{2}}{z} .
$$

The only set of PDFs which include both QCD and QED NLO corrections is the MRST2004QED [58]. As has been pointed out in [57], a consistent use of this set of PDFs requires the use of the DIS scheme for QED calculations. The coefficient functions for the DIS scheme are given by

$$
\begin{aligned}
& C_{f f}^{\mathrm{DIS}}(z)=\left[P_{f f}(z)\left(\ln \left(\frac{1-z}{z}\right)-\frac{3}{4}\right)+\frac{9+5 z}{4}\right]_{+}, \\
& C_{f \gamma}^{\mathrm{DIS}}(z)=P_{f \gamma}(z) \ln \left(\frac{1-z}{z}\right)-8 z^{2}+8 z-1 \\
& C_{\gamma f}^{\mathrm{DIS}}(z)=-C_{f f}^{\mathrm{DIS}}(z) .
\end{aligned}
$$

It should be noted that the integrated subtraction terms as they appear in MadDipole are different for processes with incoming PDF and for processes without. The introduction of the PDF does not only affect the logarithmically divergent terms, where the mass of the particle is replaced by the factorization scale, but also finite parts are different due to the coefficient functions present in the PDFs. Whether a PDF is present or not is determined automatically by MadDipole in the case of leptons or QCD particles. Any QCD particle (quark, anti-quark, gluon) is assumed to be a constituent of a hadron whereas this is certainly not true for leptons. A special case is the incoming photon, because it may come out of a hadron but could as well be a free particle. Here the user has to choose whether to use a PDF or not (see section 5).

\section{Non-collinear-safe observables}

Final state collinear photons can often be distinguished from other final state particles. For example, the energy fractions in a collinear muon-photon system can be disentangled by comparing calorimetric and tracking information, and highly energetic photons inside 
QCD jets can be identified and vetoed. Therefore, in electroweak calculations a collinear photon is not necessarily treated fully inclusively [3]. Consequently, the integration range within the cone of a photon radiated off a charged particle is not the full range of the momentum fraction of the charged particle. This momentum fraction is parametrized by the variable $z_{i j}$ for a final-final constellation and $z_{i a}$ for a final-inital one. Treating the photon not fully inclusively is now equivalent to imposing a cut on this variable. In this case, the collinear singularity associated with the photon emission off a massless fermion is not cancelled, rendering the observable non-collinear-safe. In analogy to the factorization of initial state collinear singularities into parton distributions, this singularity is absorbed into a photon fragmentation function $[20,59]$. An alternative photon isolation procedure has been proposed in the form of a dynamical cone-size [60], which would in principle shield the collinear singularity and eliminate the need for the photon fragmentation function. In practise, this criterion turns out to be difficult to implement for a finite-resolution detector. The treatment of fragmentation processes in the dipole formalism was first developed in [24] for dimensional regularization, and extended to mass regularization in [3]. We largely follow the latter paper for notation and conventions and refer to this reference for further details. Here we collect those results which were important for our implementation.

For observables with identified final state photons, the real emission matrix element and the unintegrated subtraction terms are multiplied by a step function that cuts off the integration at a given value for $z$. One therefore has

$$
\begin{aligned}
\int \mathrm{d} \Phi_{1} & {\left[\sum_{\lambda_{\gamma}}\left|\mathcal{M}_{1}\right|^{2} \Theta_{\mathrm{cut}}\left(p_{f}, k, p_{f^{\prime}},\left\{k_{n}\right\}\right)\right.} \\
& \left.-\sum_{f \neq f^{\prime}}\left|\mathcal{M}_{\mathrm{sub}, f f^{\prime}}\right|^{2} \Theta_{\mathrm{cut}}\left(z_{f f^{\prime}} \tilde{p}_{f}^{\left(f f^{\prime}\right)},\left(1-z_{f f^{\prime}}\right) \tilde{p}_{f}^{\left(f f^{\prime}\right)}, \tilde{p}_{f^{\prime}}^{\left(f f^{\prime}\right)},\left\{k_{n}\right\}\right)\right] .
\end{aligned}
$$

The integrated dipole terms are thus not integrated over $z$, but remain functions of this variable.

The MadDipole implementation usually allows for the introduction of a phase space cut, $\alpha$, to ensure that dipoles are subtracted only in the vicinity of the singular regions. In our implementation, non-collinear-safe observables can only be calculated with all $\alpha$-parameters set to one, since including the $\Theta$-function related to the $\alpha$-parameter in them would lead to very involved phase space constraints, which prevent the analytical integration of the subtraction terms.

For non-collinear safe-observables, the emitter is always in the final state, while the spectator can be in final or initial state. Both cases were derived in [3] and are summarized below. 


\section{1 final-final}

In the final-final case the integrated splitting function in mass regularization is given by

$$
\overline{\mathcal{G}}_{i j, \tau}^{(\mathrm{sub})}\left(P_{i j}^{2}, z_{i j}\right)=\frac{\bar{P}_{i j}^{4}}{2 \sqrt{\lambda_{i j}}} \int_{y_{1}\left(z_{i j}\right)}^{y_{2}\left(z_{i j}\right)} \mathrm{d} y_{i j}\left(1-y_{i j}\right) g_{i j, \tau}^{(\mathrm{sub})}\left(p_{i}, p_{j}, k\right) .
$$

For an appropriate numerical treatment the soft singularity at $z=1$ is regularized by introducing a plus distribution for $z$,

$$
\overline{\mathcal{G}}_{i j, \tau}^{(\mathrm{sub})}\left(P_{i j}^{2}, z\right)=G_{i j, \tau}^{(\mathrm{sub})}\left(P_{i j}^{2}\right) \delta(1-z)+\left[\overline{\mathcal{G}}_{i j, \tau}^{(\mathrm{sub})}\left(P_{i j}^{2}, z\right)\right]_{+} .
$$

The integration boundaries can be derived from the phase space, for details see Appendix $\mathrm{B}$ of [30]. In the limit $m_{i} \rightarrow 0$ and $m_{j}, m_{\gamma}=0$ one finds

$$
y_{1}(z)=\frac{m_{i}^{2}(1-z)}{P_{i j}^{2} z}, \quad y_{2}(z)=1 .
$$

Performing the integration leads to

$$
\begin{aligned}
& \overline{\mathcal{G}}_{i j,+}^{(\mathrm{sub})}\left(P_{i j}^{2}, z\right)=P_{f f}(z)\left[\ln \left(\frac{P_{i j}^{2} z}{m_{i}^{2}}\right)-1\right]+(1+z) \ln (1-z), \\
& \overline{\mathcal{G}}_{i j,-}^{(\mathrm{sub})}\left(P_{i j}^{2}, z\right)=1-z
\end{aligned}
$$

with

$$
P_{f f}(z)=\frac{1+z^{2}}{1-z}
$$

The case where $m_{j}$ is not zero is more involved however there is no conceptual difference to the case of a massless spectator so we do not quote the result here. It can be found in Appendix A of [3].

The derivation of the corresponding expressions in dimensional regularization starts from the dipole splitting function

$$
\frac{<\boldsymbol{V}_{i j, k}\left(z_{i} ; y\right)>}{8 \pi \alpha_{\mathrm{S}} \mu^{2 \epsilon}}=\frac{1}{\left(1-y_{i j, k}\right)}\left[\frac{2}{1-z_{i}\left(1-y_{i j, k}\right)}-1-z_{i}-\epsilon\left(1-z_{i}\right)\right],
$$

which integrates to

$$
\mathcal{V}(\epsilon, z)=-\frac{1}{\epsilon} P_{f f}(z)+P_{f f}(z) \ln (z)+\frac{z^{2}+3}{1-z} \ln (1-z)+(1-z),
$$

In the case where the spectator is massive, the splitting function takes the form

$$
\frac{<\boldsymbol{V}_{i j, k}\left(z_{j} ; y\right)>}{8 \pi \alpha_{\mathrm{S}} \mu^{2 \epsilon}}=\frac{1}{R_{i j}(y)}\left[\frac{2}{1-z_{j}\left(1-y_{i j, k}\right)}-\frac{\tilde{v}_{i j, k}}{v_{i j, k}}\left(1+z_{j}+\epsilon\left(1-z_{j}\right)\right)\right],
$$

integrating to

$$
\begin{aligned}
\mathcal{V}(\epsilon, z) & =-\frac{P_{f f}(z)}{\epsilon}+\frac{1}{z-1}\left\{z^{2}\left(-\log \left(8\left(c_{1}-c^{2}\right)\right)+\log \left(4\left(c\left(c+c_{2}\right)-c_{1} y_{2}\right)\right)+\log \left(c_{3}\right)\right)\right. \\
& +\log \left(c_{1}-c^{2}\right)-\log \left(c\left(c+c_{2}\right)-c_{1} y_{2}\right)-2\left(z^{2}-1\right) \log (c)-\log \left(c_{3}\right) \\
& \left.+2 \log \left(\left(y_{2}-1\right) z+1\right)-2 \log (1-z)+\log (2)\right\}+P_{f f}(z) \log \left(-c y_{2}(z-1) z\right)-z+1,
\end{aligned}
$$


where we used the abbreviations

$$
c=1-\mu_{k}^{2}, \quad c_{1}=1-\mu_{k}^{4}, \quad c_{2}=\sqrt{c^{2}\left(y_{2}^{2}+1\right)-2 c_{1} y_{2}}, \quad c_{3}=c_{1}-c\left(c y_{2}+c_{2}\right)
$$

and

$$
y_{2}(z)=[\xi(z)+1+\sqrt{\xi(z)(\xi(z)+2)}]^{-1}, \quad \xi(z)=\frac{\mu_{k}^{2}}{2 z(1-z)}
$$

to compactify the result.

\section{2 final-initial}

For an initial state spectator the subtraction terms depend on the variable $x_{i a}$ and are divergent for $x_{i a} \rightarrow 1$. This is taken into account by splitting off the divergent parts using the $(+)$-distribution. The correction terms for non-collinear-safe observables now come along with an additional distribution in $z$ so the the full result reads as a combination of the two.

$$
\begin{aligned}
-\frac{\bar{P}_{i a}^{2}}{2} \int_{0}^{x_{1}} \mathrm{~d} x \int_{z_{1}(x)}^{z_{2}(x)} \mathrm{d} z g_{i a, \tau}^{(\mathrm{sub})}\left(p_{i}, p_{a}, k\right) \cdots \\
=\int_{0}^{1} \mathrm{~d} x \int_{0}^{1} \mathrm{~d} z\left\{G_{i a, \tau}^{(\mathrm{sub})}\left(P_{i a}^{2}\right) \delta(1-x) \delta(1-z)+\left[\mathcal{G}_{i a, \tau}^{(\mathrm{sub})}\left(P_{i a}^{2}, x\right)\right]_{+} \delta(1-z)\right. \\
\left.+\left[\overline{\mathcal{G}}_{i a, \tau}^{(\mathrm{sub})}\left(P_{i a}^{2}, z\right)\right]_{+} \delta(1-x)+\left[\bar{g}_{i a, \tau}^{(\mathrm{sub})}(x, z)\right]_{+}^{(x, z)}\right\} \cdots
\end{aligned}
$$

with

$$
\begin{aligned}
\bar{g}_{i a,+}^{(\mathrm{sub})}(x, z) & =\frac{1}{1-x}\left(\frac{2}{2-x-z}-1-z\right), \quad \bar{g}_{i a,-}^{(\mathrm{sub})}(x, z)=0, \\
\overline{\mathcal{G}}_{i a,+}^{(\mathrm{sub})}\left(P_{i a}^{2}, z\right) & =P_{f f}(z)\left[\ln \left(\frac{-P_{i a}^{2} z}{m_{i}^{2}}\right)-1\right]-\frac{2 \ln (2-z)}{1-z}+(1+z) \ln (1-z), \\
\overline{\mathcal{G}}_{i a,-}^{(\mathrm{sub})}\left(P_{i a}^{2}, z\right) & =1-z .
\end{aligned}
$$

The first contribution, $\bar{g}_{i a, \tau}^{(\mathrm{sub})}(x, z)$ is identical in mass regularization and dimensional regularization.

To evaluate the equivalent expression of $\overline{\mathcal{G}}_{i a, \tau}^{\text {(sub) }}\left(P_{i a}^{2}, z\right)$ in dimensional regularization we use the splitting function and phase space given in Eqns. (5.39) and (5.48) of [24] and find

$$
\mathcal{V}(\epsilon, z)=-\frac{P_{f f}(z)}{\epsilon}+P_{f f}(z) \log (z)+\frac{z^{2}+3}{1-z} \log (1-z)-2 \frac{\log (2-z)}{1-z}+1-z .
$$

\subsection{Factorization of the collinear final state singularity}

The integrated dipole factors contain a collinear singularity. For fully inclusive observables, the dipole factors would be integrated over the photon momentum fraction $z$ and added with the virtual photon corrections, rendering the result finite. Since non-collinear-safe observables explicitly require (or veto) the final state photon, these cancellations no longer take place. In the case of photon radiation off massive fermions, this results in a large logarithm in the fermion mass, which is present in the above mass-regularization results. 
For massless fermions, especially for light quarks, this turns into a collinear singularity, and one has to include a contribution from the fragmentation of a final state fermion into a photon-fermion system of low invariant mass. The quark-to-photon fragmentation function (which depends on the photon momentum fraction $(1-z)$ ) is an empirically determined quantity $[20,59,61,62]$. It contains a counterterm for the collinear singularity, whose explicit form depends on the regularization scheme. The counterterm is accounted for by adding the bare fragmentation function to the integrated dipole terms. In mass regularization, the bare fragmentation function reads [63]:

$$
D_{q \rightarrow \gamma}^{\mathrm{bare}, \mathrm{MR}}(1-z)=D_{q \rightarrow \gamma}\left(1-z, \mu_{F}\right)+\frac{\alpha Q_{q}^{2}}{2 \pi} P_{f f}(z)\left[\ln \left(\frac{m_{q}^{2}}{\mu_{F}^{2}}(1-z)^{2}\right)+1\right] \text {, }
$$

while the corresponding expression in dimensional regularization is [20]:

$$
D_{q \rightarrow \gamma}^{\mathrm{bare}, \mathrm{DR}}(1-z)=D_{q \rightarrow \gamma}\left(1-z, \mu_{F}\right)+\frac{\alpha Q_{q}^{2}}{2 \pi} \frac{1}{\epsilon}\left(\frac{4 \pi \mu^{2}}{\mu_{F}^{2}}\right)^{\epsilon} \frac{1}{\Gamma(1-\epsilon)} P_{f f}(z) .
$$

It can be seen that the sum of the integrated dipole terms and the bare fragmentation function is independent on the regularization scheme. The scale-dependent non-perturbative fragmentation function $D_{q \rightarrow \gamma}\left(1-z, \mu_{F}\right)$ has to be determined from data.

Our implementation offers the possibility to include the fragmentation function. For details see section 5 .

\section{Implementation and how to use it}

The installation and running of the new package is identical to the QCD version:

1. Download the MadDipole package (version 4.4.35 or later), MG_ME_DIP_V4.4.?? .tar.gz, from one of the MadGraph websites, e.g., http://madgraph.hep.uiuc.edu/.

2. Extract and run make in the MadGraphII directory.

3. Copy the Template directory into a new directory, e.g., MyProcDir to ensure that you always have a clean copy of the Template directory.

4. Go to the new MyProcDir directory and specify your process in the file ./Cards/proc_card.dat. This is the $(n+1)$-particle process you require the subtraction term for. In this file there is also a variabe DipolePhotonPdf which is set to TRUE by default. If so it is assumed that initial state photons are coming from a hadron and therefore a PDF is used.

5. Running ./bin/newprocess_qed generates the code for the $(n+1)$-particle matrix element and for all dipole terms and their integrated versions. After running this you will find a newly generated directory ./SubProcesses/P0_yourprocess (e.g., ./SubProcesses/PO_e+e-_uuxa) which contains all required files. 
In the ./SubProcesses/P0_yourprocess directory all the files relevant to that particular subprocesses are generated. In particular this includes the $(n+1)$ particle matrix elements in the file matrix.f and the dipoles in the files dipolqed???.f, where ??? stands for a number starting from 001. Furthermore the directory has two files, dipolsumqed.f and intdipolesqed.f, where the sum of the dipoles and their integrated versions are calculated, respectively.

The routines contained in intdipolesqed.f which calculate the integrated subtraction terms have a slightly different syntax compared to the QCD version so we give some more details here. As for QCD the file contains two routines

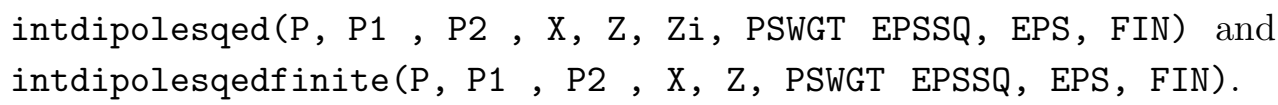

The difference is that the routines need three phase space points as input variables. This is due to the problem explained in section 1, that in the absence of a PDF the reduced matrix element depends on the longitudinal momentum fraction $x$ (here named as $\mathrm{Z}$ ). Therefore the phase space point $\mathrm{P}(0: 3$, nexternal $)$ denotes the point in an $n$-particle phase space with $x=1$. The next variable, P1 ( $0: 3$, nexternal) denotes the phase space point with an initial state radiation from the first incoming particle, which means its momentum is given by $x \cdot p_{1}$ where $p_{1}$ is the full first momentum as it appears in the first phase space point. The third phase space point is the same as second but with the radiation off the second incoming particle.

These phase space points have to be provided by the user. In the case where there are PDFs present for both incoming particles we use the same method as in QCD, namely shifting the dependence of the matrix element on the momentum fraction $x$ into the PDF. In that case only the first phase space point $\mathrm{P}(0: 3$, nexternal) has to be provided, the others are not used.

The second difference to QCD is the appearence of the variable $\mathrm{Zi}$. It is only needed for non-collinear-safe observables (see section 4) and denotes the integration variable $z$ as defined in that context. We assume that the integration over $\mathrm{Zi}$ is carried out numerically and therefore this variable has to be provided by the user.

Several important parameters are included in the file dipole.inc which need to be set by the user.

The first parameter decides which regularization scheme should be used. It can be either DREG or MREG which stands for dimensional regularization and mass regularization respectively. Note that in the case where the reduced matrix element contains leptons in the initial state, one has to choose mass regularization.

The second parameter is a logical variable photonpdf which determines whether a PDF should be used for initial state photons. Whether this parameter is true or false per default is determined by the choice the user made in param_card.dat. If the according variable in the parameter card is set to true, this variable in dipole.inc will be set to true au- 
tomatically when the code is generated. So for consistency this parameter should not be changed by the user. The next variable is a real array massint which contains the particle masses and which have to be given by the user. More precisely, what is required are the masses of the particles as they appear in the integrated subtraction terms. As an example we can take $e^{+} e^{-} \rightarrow t \bar{t} \gamma$. For the real emission matrix element and for the unintegrated dipoles one would usually treat the electron as a massless particle whereas the top-quark would be treated as a massive particle. The integrated subtraction terms however require an electron mass to regulate the infrared singularities. These regulator masses are declared in the array. So in this example one would fill the first two entries with the electron mass, and the next two entries would be the top mass. A photon should be treated massless in this array, so the last entry would be zero. All units are in $G e V$. Per default all entries are set to one to ensure a finite result.

The next parameter is the photon mass parameter, which is also needed to regulate logarithmic divergencies. By default it is set to unity so that all logarithms containing the photon mass vanish.

The following parameter is a logical variable ncs. If set to true it calculates the correction terms for non-collinear safe observables. This requires of course that the user has to provide appropriate values for the variable $\mathrm{Zi}$ described above.

The last parameter is given by a logical variable photonfrag which is set to true by default. If set to true the second terms on the right hand side of Eqns. (4.16/4.17) are added to the integrated dipoles and a template function is called which can be used by the user to provide the non-perturbative parts of the fragmentation function, given by the first term on the right hand side of Eqns. (4.16/4.17). This template function is described in section 5.1 .

\subsection{Implementation of non-collinear-safe case}

The calculation of non-collinear-safe observable requires to introduce additional cuts which act on the matrix element as well as on the subtraction terms, as in Eq. (4.1). In MadDipole these cuts can be imposed using the predefined cut routines, no additional routines are introduced. The correct handling of the $\Theta$-function has to be done by the user.

Also the integrated subtraction terms receive additional cuts in form of a $\Theta$-function. For the final-final case, the integrated subtraction terms are therefore of the form

$$
\begin{aligned}
\int \mathrm{d} \Phi_{1}\left|\mathcal{M}_{\mathrm{sub}, i j}\left(\Phi_{1} ; \kappa_{i}\right)\right|^{2}= & -\frac{\alpha}{2 \pi} Q_{i} \sigma_{i} Q_{j} \sigma_{j} \int \mathrm{d} \tilde{\Phi}_{0, i j} \int_{0}^{1} \mathrm{~d} z \\
& \times\left\{G_{i j, \tau}^{(\mathrm{sub})}\left(P_{i j}^{2}\right) \delta(1-z)+\left[\overline{\mathcal{G}}_{i j, \tau}^{(\mathrm{sub})}\left(P_{i j}^{2}, z\right)\right]_{+}\right\} \\
& \times\left|\mathcal{M}_{0}\left(\tilde{p}_{i}, \tilde{p}_{j} ; \tau \kappa_{i}\right)\right|^{2} \Theta_{\mathrm{cut}}\left(p_{i}=z \tilde{p}_{i}, k=(1-z) \tilde{p}_{i}, \tilde{p}_{j},\left\{k_{n}\right\}\right),
\end{aligned}
$$

The parts that are $z$-dependent are the $(+)$-distribution and the $\Theta$-function. This means that this $\Theta$-function regularizes the singularity contained in the $(+)$-distribution at $z=1$. We therefore need to evaluate the step function at two points, $z \neq 1$ and $z=1$. As this can not be done within the predefined cut routines, we introduce an additional logical variable 
- thetacut $(P, z i)$

which can be either true or false. Its arguments are the phase space point and the variable $z$. As MadDipole uses a variable $z$ already to denote the momentum fraction in the context of initial state radiation we rename this variable to $z i$. In the same way as for the 'normal' cut routine MadDipole provides an empty dummy routine in which the user can specify the desired cuts. MadDipole then takes care that it is evaluated at the two different points and combined with the subtraction terms according to (5.1). The template of this function is included in the file intdipolesqed.f.

In the same file we also provide a template that can be used for calculating the nonperturbative parts of the quark-to-photon fragmentation function as described in the previous section. The template is given by the real function

- $\operatorname{DFRAG}(z i)$

where the only input variable is $z$. Here the user should provide a call to the set of fragmentation functions of his choice. The result will then be added automatically to the integrated dipole terms. Per default, this routine returns zero so no non-perturbative terms are added.

\section{Checks}

Several checks have been performed to test the correctness of the different parts of the implementation. A first check of the unintegrated dipoles is of course to check the limits, i.e. the fact that the ratio of real emission matrix element over the sum of all subtraction terms,

$$
\frac{\left|\mathcal{M}_{\text {real }}\right|^{2}}{\sum \mathcal{D}} \rightarrow 1
$$

when one approaches a soft- and/or collinear limit. This could easily be done using the already existing check program which is part of MadDipole (see [53] for details).

This alone is not a sufficient test. Furthermore we compared results for single phase space points against existing results for the processes $e^{+} e^{-} \rightarrow 4 f$ with RacoonWW [64-66], $e^{-} \gamma \rightarrow e^{-} \mu^{+} \mu^{-}[3]$ and $e^{+} e^{-} \rightarrow t \bar{t} H[67,68]$. Thus all different splittings could be tested. This has been done for both the unintegrated dipoles as well as for the integrated ones.

The aim of this package is to provide the necessary subtraction terms for single phase space points without the phase space integration. In particular the user is not restricted to MadEvent for the integration but can use MadDipole as a stand alone package and implement it in a completely different framework. A pointwise agreement with existing results should therefore be considered sufficient to test the implementation. The calculation of complete cross sections (with virtual corrections from other packages) is beyond the scope of this work.

Furthermore we repeated the checks on the dependence of the cut parameter $\alpha$ as described in [55]. Although already being checked in the QCD version of MadDipole additional checks 


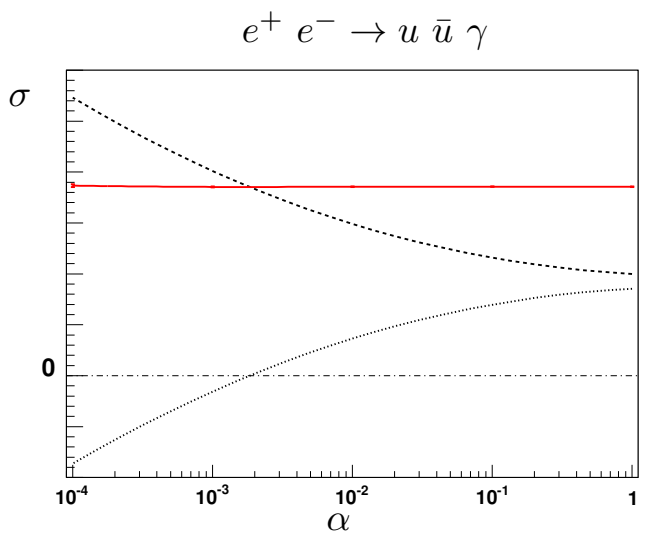

(a)

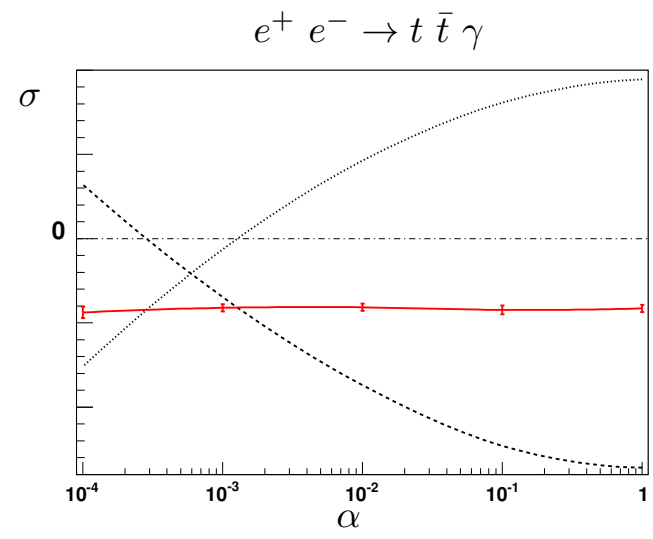

(b)

Figure 1: Dependence of the cross section on the cut parameter $\alpha$. All four $\alpha$-parameters (finalfinal, final-initial, initial-final, initial-initial) are varied simultaneously. The dashed line denotes the real emission matrix element where the dipoles have been subtracted. The dotted line is the contribution from the finite parts of the integrated dipoles. The solid red line is the sum of the two contributions. As this is by no means a physical meaningful quantity we do not give actual numbers for the cross section. For the sum, the error bars of the Monte Carlo integration are added.

were necessary because of two reasons. First of all the final-final dipole splitting function $f^{*} \rightarrow f \gamma$ is conventionally normalized differently than in QCD, which made it necessary to calculate the correction terms (see appendix). On the other hand having leptons in the initial state made it necessary to incorporate the concept of calculating amplitudes at different points $(x=1$ and $x \neq 1)$ which is not needed for QCD processes. Checking the independence of the total result on the phase space cut parameter provides a valuable test for this part of the implementation.

In Fig. 6 we show the dependence of the $\alpha$-parameter for two processes, $e^{+} e^{-} \rightarrow u \bar{u} \gamma$ and $e^{+} e^{-} \rightarrow t \bar{t} \gamma$. These two processes contain the final-final splittings different from QCD and they also contain initial state radiation off leptons. What the plots show is the cross section as a function of the cut parameter. Actually, each splitting (final-final, final-initial, initialfinal and initial-initial) has its own $\alpha$-parameter which can be adjusted independently from each other. In this plot we change them simultaneously so that their values are always equal.

The dashed line shows the cross section of the real emission matrix element where the dipoles have been subtracted so that the difference is finite. The dotted line shows the cross section of the finite parts of the integrated dipoles. Summing both contributions leads to the solid red line which shows an independence on the cut parameter to a very good accuracy over several orders of magnitude.

\section{Conclusions}

The MadDipole package automatically generates unintegrated and integrated subtraction terms required for NLO calculations. Previously, it provided all dipole subtraction terms for QCD radiation $[53,55]$. In this paper, we extended MadDipole to include photonic 
radiation. The implementation is provided both in dimensional regularization and mass regularization. We include counterterms for the QED corrections to the parton distribution functions, and allow for subtraction terms for non-collinear-safe observables, i.e. with identified final state photons.

With the photonic radiation now included in MadDipole, the MadGraph/MadEvent framework can now be used for electroweak real radiation corrections to collider observables. This development provides an important step towards the full automation of electroweak NLO corrections.

\section{Acknowledgments}

We are very grateful to Stefan Dittmaier and Marcus Weber for numerous valuable discussions, support, providing results and performing comparisons. We also thank Rikkert Frederix and Tim Stelzer for their help to make the code public. This work was supported by the U.S. Department of Energy under contract No. DE-FG02-91ER40677 and by the Swiss National Science Foundation (SNF) under contract 200020-126691.

\section{A. Final-final $f \rightarrow f+\gamma$ dipole in dimensional regularization}

The final-final dipole factor for the radiation of a photon off a fermion is conventionally normalized differently from the corresponding QCD case. It is therefore documented in detail here. For the case where a photon is radiated from a massless fermion the auxiliary function is given by (Eqn. (3.1) of [30])

$$
\begin{aligned}
& g_{i j,+}^{(\mathrm{sub})}\left(p_{i}, p_{j}, k\right)=\frac{1}{\left(p_{i} k\right)\left(1-y_{i j}\right)}\left[\frac{2}{1-z_{i j}\left(1-y_{i j}\right)}-1-z_{i j}\right] \\
& g_{i j,-}^{(\mathrm{sub})}\left(p_{i}, p_{j}, k\right)=0 .
\end{aligned}
$$

In this notation $k$ is the momentum of the photon, $p_{i}$ and $p_{j}$ are the momenta of emitter and spectator. The variables $y_{i j}$ and $z_{i j}$ correspond to the variables $y$ and $z$ of the QCD case. Note that there is an additional factor of $\frac{1}{1-y_{i j}}$ present in this case.

The integrated term in dimensional regularization is obtained along the lines of the CataniSeymour approach [24].

We want to use dimensional regluarisation therefore we are now following the CataniSeymour approach. In that language the integrated term is given by Eqns. (5.27,5.28) of [24]:

$$
\mathcal{V}_{i j, k}=\int\left[d p_{i}\left(\widetilde{p}_{i j}, \widetilde{p}_{k}\right)\right] \frac{1}{2 p_{i} \cdot p_{j}}<\boldsymbol{V}_{i j, k}>\equiv \frac{\alpha_{\mathrm{S}}}{2 \pi} \frac{1}{\Gamma(1-\epsilon)}\left(\frac{4 \pi \mu^{2}}{2 \widetilde{p}_{i j} \widetilde{p}_{k}}\right)^{\epsilon} \mathcal{V}_{i j}(\epsilon)
$$

and

$$
\mathcal{V}_{i j}(\epsilon)=\int_{0}^{1} d \tilde{z}_{i}\left(\tilde{z}_{i}\left(1-\tilde{z}_{i}\right)\right)^{-\epsilon} \int_{0}^{1} \frac{d y}{y}(1-y)^{1-2 \epsilon} y^{-\epsilon} \frac{<\boldsymbol{V}_{i j, k}\left(\tilde{z}_{i} ; y\right)>}{8 \pi \alpha_{\mathrm{S}} \mu^{2 \epsilon}}
$$


The transition can be made by replacing

$$
\frac{<\boldsymbol{V}_{i j, k}\left(\tilde{z}_{i} ; y\right)>}{8 \pi \alpha_{\mathrm{S}} \mu^{2 \epsilon}} \rightarrow \frac{1}{\left(1-y_{i j}\right)}\left[\frac{2}{1-z_{i j}\left(1-y_{i j}\right)}-1-z_{i j}-\epsilon\left(1-z_{i j}\right)\right] .
$$

After integration and expanding in $\epsilon$ we find

$$
\mathcal{V}_{\gamma f}(\epsilon)=\frac{1}{\epsilon^{2}}+\frac{3}{2 \epsilon}+\left(\frac{7}{2}-\frac{\pi^{2}}{6}\right)+O\left(\epsilon^{1}\right)
$$

The massive case is more involved. We decompose the integral into an eikonal and a collinear part,

$$
I_{\gamma f, k}\left(\mu_{j}, \mu_{k} ; \epsilon\right)=\left[2 I^{\mathrm{eik}}\left(\mu_{j}, \mu_{k} ; \epsilon\right)+I_{\gamma f, k}^{\mathrm{coll}}\left(\mu_{j}, \mu_{k} ; \epsilon\right)\right],
$$

where we define the eikonal integral in analogy to Eq. (5.26) of [25]

$$
\frac{\alpha_{\mathrm{s}}}{2 \pi} \frac{1}{\Gamma(1-\epsilon)}\left(\frac{4 \pi \mu^{2}}{Q^{2}}\right)^{\epsilon} I^{\mathrm{eik}}\left(\mu_{j}, \mu_{k} ; \epsilon\right)=\int\left[\mathrm{d} p_{i}\left(\widetilde{p}_{i j}, \widetilde{p}_{k}\right)\right] \frac{1}{2 p_{i} p_{j}} \frac{1}{R_{i j}(y)} \frac{8 \pi \mu^{2 \epsilon} \alpha_{\mathrm{s}}}{1-\tilde{z}_{j}\left(1-y_{i j, k}\right)} .
$$

The difference to the CS dipoles is given by the additional factor $R_{i j}(y)$, which is defined in Eq. (4.3) of [30]

$$
R_{i j}(y)=\frac{\sqrt{\left(2 m_{j}^{2}+\bar{P}_{i j}^{2}-\bar{P}_{i j}^{2} y\right)^{2}-4 P_{i j}^{2} m_{j}^{2}}}{\sqrt{\lambda_{i j}}},
$$

with

$$
P_{i j}=p_{i}+p_{j}+k, \quad \bar{P}_{i j}^{2}=P_{i j}^{2}-m_{i}^{2}-m_{j}^{2}-m_{\gamma}^{2}, \quad \lambda_{i j}=\lambda\left(P_{i j}^{2}, m_{i}^{2}, m_{j}^{2}\right) .
$$

Performing the integration we find for the eikonal integral

$$
\begin{gathered}
I^{\mathrm{eik}}\left(\mu_{j}, \mu_{k} ; \epsilon\right)=\frac{1}{\epsilon} \frac{\ln \rho_{j}}{\tilde{v}}+\frac{1}{\tilde{v}}\left\{\operatorname{Li}_{2}\left(\frac{\left(c_{j}-\tilde{v}+1\right)\left(c_{k}-\tilde{v}+1\right)}{\left(c_{j}+c_{k}+2\right)\left(c_{k}-y_{+}+1\right)-c_{2}}\right)+\operatorname{Li}_{2}\left(\frac{\left(c_{j}+c_{k}+2\right)\left(c_{k}-y_{+}+1\right)-c_{2}}{(c+\tilde{v}+1)\left(c_{k}+\tilde{v}+1\right)}\right)\right. \\
+2 \ln (\rho) \ln \left(\left(c_{j}+c_{k}+2\right)\left(c_{k}-y_{+}+1\right)-c_{2}\right)+\frac{1}{2} \ln ^{2}\left(\frac{\left(c_{j}+c_{k}+2\right)\left(-c_{k}+y_{+}-1\right)+c_{2}}{\left(c_{j}-\tilde{v}+1\right)\left(-c_{k}+\tilde{v}-1\right)}\right) \\
+\ln (2 \tilde{v}) \ln \left(\frac{\left(c_{j}+c_{k}+2\right)\left(c_{k}-\tilde{v}+1\right)-c_{2}}{c_{j}-\tilde{v}+1}\right)+\left(\ln \left(c_{j}-\tilde{v}+1\right) \ln \left(\frac{c_{k}+\tilde{v}+1}{\rho_{j}^{2}}\right)\right. \\
\left.-\ln \left(2 \tilde{v}\left(c_{j}-\tilde{v}+1\right)\right) \ln \left(c_{k}-\tilde{v}+1\right)-\ln (2 \tilde{v}) \ln \left(c_{j}-\tilde{v}+1\right)\right)+\operatorname{Li}_{2}\left(\frac{c_{j}-\tilde{v}+1}{c_{j}+y_{+}+1}\right) \\
+\operatorname{Li}_{2}\left(\frac{c_{j}+y_{+}+1}{c_{j}+\tilde{v}+1}\right)+\ln \left(c_{j}+\tilde{v}+1\right) \ln \left(\frac{2 \tilde{v}}{\rho_{j}^{2}}\right)+2 \ln \left(\rho_{j}\right) \ln \left(c_{j}+y_{+}+1\right) \\
+\frac{1}{2} \ln ^{2}\left(\frac{c_{j}+y_{+}+1}{c_{j}-\tilde{v}+1}\right)-\operatorname{Li}_{2}\left(\frac{c_{k}-\tilde{v}+1}{c_{k}-y_{+}+1}\right)-\operatorname{Li}_{2}\left(\frac{c_{k}-y_{+}+1}{c_{k}+\tilde{v}+1}\right)-2 \ln \left(\rho_{k}\right) \ln \left(c_{k}-y_{+}+1\right) \\
+\ln \left(\rho_{j}\right) \ln \left(\frac{\left(\tilde{v}-y_{+}\right)^{2}\left(\tilde{v}+y_{+}\right)^{2}}{4 \rho_{j}^{2} \tilde{v}^{2}\left(c_{k}-y_{+}+1\right)^{2}}\right)-\frac{1}{2} \ln { }^{2}\left(\frac{c_{k}-y_{+}+1}{c_{k}-\tilde{v}+1}\right)-\operatorname{Li}_{2}\left(\rho^{2}\right)+\operatorname{Li}_{2}\left(\rho_{k}^{2}\right) \\
\left.-\operatorname{Li}_{2}\left(\rho_{j}^{2}\right)-\frac{\pi^{2}}{6}+\operatorname{Li}_{2}\left(-\frac{2 \tilde{v}}{\rho_{j}^{2}\left(c_{j}+\tilde{v}+1\right)}\right)-\ln \left(\rho_{j}\right) \ln \left(\frac{2 y_{+}^{2} \sqrt{\lambda}}{c \rho_{j} \tilde{v}}\right)\right\}+\mathcal{O}(\epsilon),
\end{gathered}
$$

where we used the following abbreviations:

$$
\begin{array}{rlrl}
\sqrt{\lambda} & =\sqrt{\lambda\left(1, \mu_{j}^{2}, \mu_{k}^{2}\right)} & , \rho_{n}\left(\mu_{j}, \mu_{k}\right)=\sqrt{\frac{1-\tilde{v}_{i j, k}+2 \mu_{n}^{2} /\left(1-\mu_{j}^{2}-\mu_{k}^{2}\right)}{1+\tilde{v}_{i j, k}+2 \mu_{n}^{2} /\left(1-\mu_{j}^{2}-\mu_{k}^{2}\right)}} \quad(n=j, k), \\
\tilde{v} & =\tilde{v}_{i j, k}, & \rho=\sqrt{\frac{1-\tilde{v}_{i j, k}}{1+\tilde{v}_{i j, k}}} &
\end{array}
$$


and

$$
y_{+}=1-\frac{2 \mu_{k}\left(1-\mu_{k}\right)}{1-\mu_{i}^{2}-\mu_{j}^{2}-\mu_{k}^{2}}
$$

is the upper limit of the $y$ integration. Furthermore we define for brevity

$$
c_{j}=\frac{2 \mu_{j}^{2}}{1-\mu_{j}^{2}-\mu_{k}^{2}}, \quad c_{k}=\frac{2 \mu_{k}^{2}}{1-\mu_{j}^{2}-\mu_{k}^{2}}, \quad c_{2}=\frac{4 \mu_{k}^{2}}{\left(1-\mu_{j}^{2}-\mu_{k}^{2}\right)^{2}} .
$$

The contribution from the collinear integral is simpler and we find

$$
\begin{gathered}
I_{\gamma f, k}^{\text {coll }}\left(\mu_{f}, \mu_{k} ; \epsilon\right)=\frac{1}{\epsilon}+\frac{1}{2}\left(-4 \log \left(-\mu_{j}^{2}+\mu_{k}^{2}-2 \mu_{k}+1\right)\right. \\
\left.+\frac{\mu_{j}^{2}}{\left(\mu_{k}-1\right)^{2}}+3\right)+\log \left(\mu_{j}\left(1-\mu_{k}\right)\right)+\mathcal{O}(\epsilon) .
\end{gathered}
$$

The results in dimensional regularization can be compared with the results in mass regularization given in Eq. (4.10) of [30]. Comparing the two results numerically we find full agreement.

As the limit $\epsilon \rightarrow 0$ does not commute with the limit of vanishing masses we also give the explicit result for the case when one mass is set to zero. In the case of a vanishing emitter mass we find

$$
\begin{gathered}
I^{\text {eik }} \quad\left(0, \mu_{k} ; \epsilon\right)=\frac{1}{2 \epsilon^{2}}-\frac{\ln \left(1-\mu_{k}^{2}\right)}{\epsilon}+2 \operatorname{Li}_{2}\left(\frac{1}{\mu_{k}+1}\right)+\ln \left(\frac{1}{\mu_{k}}-1\right) \ln \left(1-\mu_{k}\right) \\
+\ln \left(\mu_{k}+1\right)\left(4 \ln \left(1-\mu_{k}\right)-3 \ln \left(\mu_{k}\right)+2 \ln \left(\mu_{k}+1\right)\right)-\frac{7 \pi^{2}}{12}+\mathcal{O}(\epsilon),
\end{gathered}
$$

whereas for vanishing spectator mass we find

$$
\begin{aligned}
I^{\mathrm{eik}}\left(\mu_{j}, 0 ; \epsilon\right) & =\frac{\ln \left(\mu_{j}\right)}{\epsilon}+2 \operatorname{Li}_{2}\left(1-\frac{1}{\mu_{j}^{2}}\right)-\operatorname{Li}_{2}\left(\mu_{j}^{2}\right)-4 \log \left(1-\mu_{j}^{2}\right) \log \left(\mu_{j}\right) \\
& +3 \log ^{2}\left(\mu_{j}\right)+\frac{\pi^{2}}{6}+\mathcal{O}(\epsilon)
\end{aligned}
$$

The corresponding terms from the collinear integral read as

$$
I_{\gamma f, k}^{\text {coll }}\left(0, \mu_{k} ; \epsilon\right)=\frac{3}{2 \epsilon}+\frac{7}{2}-3 \log \left(1-\mu_{k}\right)+\mathcal{O}(\epsilon)
$$

and

$$
I_{\gamma f, k}^{\mathrm{coll}}\left(\mu_{j}, 0 ; \epsilon\right)=\frac{1}{\epsilon}+\frac{1}{2}\left\{\mu_{j}^{2}-4 \log \left(1-\mu_{j}^{2}\right)+2 \log \left(\mu_{j}\right)+3\right\}+\mathcal{O}(\epsilon) .
$$

\section{A.1 $\alpha$-cut parameter}

The idea and the use of the $\alpha$-parameter is described in detail in [69,70]. As the final-final splitting function for the splitting $f^{*} \rightarrow f \gamma$ is different from the one in QCD the results were not present in MadDipole so far.

These correction terms only affect finite terms and have no influence on singular terms which means they can be calculated either in the mass regularization approach or in the 
dimensional regularization approach. The correction terms are the same so one can use the method that appears to be more suitable.

The general form of the correction terms in the language of dimensional regularization is given by

$$
I_{\gamma f, k}\left(\mu_{j}, \mu_{k} ; \epsilon, \alpha\right)=I_{\gamma f, k}\left(\mu_{j}, \mu_{k} ; \epsilon\right)+\Delta I_{\gamma f, k}\left(\mu_{j}, \mu_{k} ; \alpha\right) .
$$

The massless case is straightforward and we find

$$
\Delta I_{\gamma f, k}(0,0 ; \alpha)=-2 \operatorname{Li}_{2}(1-\alpha)-\log ^{2}(\alpha)-\frac{3 \log (\alpha)}{2} .
$$

In the massive case we calculate eikonal integral and collinear integral seperately. For the case where both emitter and spectator are massive we find

$$
\begin{aligned}
\Delta I^{\mathrm{eik}} & \left(\mu_{j}, \mu_{k}, \alpha\right)=\frac{1}{2 v}\left\{2 \operatorname{Li}_{2}\left(\frac{v^{2}+c+c c_{k}+c_{k}-\left(c+c_{k}+2\right) y_{\alpha}+1}{(c+v+1)\left(c_{k}+v+1\right)}\right)\right. \\
& -2 \operatorname{Li}_{2}\left(\frac{v^{2}+c+c c_{k}+c_{k}-\left(c+c_{k}+2\right) y_{+}+1}{(c+v+1)\left(c_{k}+v+1\right)}\right)+2 \operatorname{Li}_{2}\left(\frac{(c-v+1)\left(-c_{k}+v-1\right)}{\left(c+c_{k}+2\right)\left(y_{\alpha}-1\right)}\right) \\
& -2 \operatorname{Li}_{2}\left(\frac{(c-v+1)\left(-c_{k}+v-1\right)}{\left(c+c_{k}+2\right)\left(y_{+}-1\right)}\right)-2 \operatorname{Li}_{2}\left(\frac{c-v+1}{c+y_{+}+1}\right)-2 \operatorname{Li}_{2}\left(\frac{c+y_{+}+1}{c+v+1}\right) \\
& -2 \log \left(\frac{v+y_{\alpha}}{v-y_{\alpha}}\right) \log \left(\frac{\left(c+y_{\alpha}+1\right)\left(c_{k}-y_{\alpha}+1\right)}{-y_{\alpha}\left(c+c_{k}+2\right)+c c_{k}+c+c_{k}+v^{2}+1}\right) \\
& +2 \log \left(\frac{v+y_{+}}{v-y_{+}}\right) \log \left(\frac{\left(c+y_{+}+1\right)\left(c_{k}-y_{+}+1\right)}{-y_{+}\left(c+c_{k}+2\right)+c c_{k}+c+c_{k}+v^{2}+1}\right) \\
& +\left(\log \left(1-y_{\alpha}\right)-\log \left(1-y_{+}\right)\right)\left(-2 \log \left((c+v+1)\left(c_{k}+v+1\right)\right)+2 \log \left(c+c_{k}+2\right)\right. \\
& \left.+\log \left(1-y_{\alpha}\right)+\log \left(1-y_{+}\right)\right)+2 \operatorname{Li}_{2}\left(\frac{c-v+1}{c+y_{\alpha}+1}\right)+2 \operatorname{Li}_{2}\left(\frac{c+y_{\alpha}+1}{c+v+1}\right) \\
& -\left(\log \left(c+y_{\alpha}+1\right)-\log \left(c+y_{+}+1\right)\right)\left(2 \log (c+v+1)-\log \left(c+y_{\alpha}+1\right)-\log \left(c+y_{+}+1\right)\right) \\
& +\left(\log \left(c_{k}-y_{\alpha}+1\right)-\log \left(c_{k}-y_{+}+1\right)\right)\left(2 \log \left(c_{k}+v+1\right)-\log \left(c_{k}-y_{\alpha}+1\right)-\log \left(c_{k}-y_{+}+1\right)\right) \\
& \left.-2 \operatorname{Li}_{2}\left(\frac{c_{k}-v+1}{c_{k}-y_{\alpha}+1}\right)-2 \operatorname{Li}_{2}\left(\frac{c_{k}-y_{\alpha}+1}{c_{k}+v+1}\right)+2 \operatorname{Li}_{2}\left(\frac{c_{k}-v+1}{c_{k}-y_{+}+1}\right)+2 \operatorname{Li}_{2}\left(\frac{c_{k}-y_{+}+1}{c_{k}+v+1}\right)\right\}
\end{aligned}
$$

with

$$
y_{\alpha}=\sqrt{\left(c_{k}(1-\alpha)+\left(\sqrt{c_{2}}-1\right) \alpha+1\right)^{2}-c_{2}}+\alpha\left(c_{k}-\sqrt{c_{2}}+1\right) .
$$

For a massless emitter and a massive spectator the correction term is given by

$$
\begin{aligned}
& \Delta I^{\mathrm{eik}}\left(0, \mu_{k}, \alpha\right)=\log \left(1-y_{\alpha}\right) \log \left(-\frac{4}{\left(\mu_{k}^{2}-1\right)\left(y_{\alpha}+1\right)^{2}}\right) \log \left(y_{\alpha}+1\right) \log \left(-\left(\mu_{k}^{2}-1\right)\left(y_{\alpha}+1\right)\right) \\
& \quad+2 \operatorname{Li}_{2}\left(\frac{1}{\mu_{k}+1}\right)-\log \left(2-2 \mu_{k}\right) \log \left(\frac{2}{\mu_{k}+1}\right)+\log \left(\frac{2 \mu_{k}}{\mu_{k}+1}\right) \log \left(\frac{2}{\mu_{k}+1}-1\right) \\
& \quad-2 \operatorname{Li}_{2}\left(\frac{y_{\alpha}+1}{2}\right)
\end{aligned}
$$


with

$$
y_{\alpha}=\frac{\alpha\left(1-\mu_{k}\right)+\sqrt{(\alpha-1)\left(\left(\mu_{k}-1\right)^{2} \alpha-\left(\mu_{k}+1\right)^{2}\right)}}{\mu_{k}+1} .
$$

Finally for the case of a massive emitter and a massless spectator we find

$$
\begin{aligned}
& \Delta I^{\mathrm{eik}}\left(\mu_{j}, 0, \alpha\right)=-\operatorname{Li}_{2}\left(\alpha-\frac{\alpha}{\mu_{j}^{2}}\right)-\operatorname{Li}_{2}\left(-\alpha \mu_{j}^{2}+\mu_{j}^{2}+\alpha\right)+\operatorname{Li}_{2}\left(1-\frac{1}{\mu_{j}^{2}}\right) \\
& -\log \left(\left(\mu_{j}^{2}-1\right)(\alpha-1)\right) \log \left(\mu_{j}^{2}(-\alpha)+\mu_{j}^{2}+\alpha\right)+2 \log \left(\mu_{j}\right) \log (\alpha)+\frac{\pi^{2}}{6} .
\end{aligned}
$$

In the same way as for the eikonal integral we take the $\alpha$-dependence of the collinear integral into account by writing

$$
I_{\gamma f, k}^{\text {coll }}\left(\mu_{j}, \mu_{k} ; \epsilon, \alpha\right)=I_{\gamma f, k}^{\text {coll }}\left(\mu_{j}, \mu_{k} ; \epsilon\right)+\Delta I_{\gamma f, k}^{\text {coll }}\left(\mu_{j}, \mu_{k} ; \alpha\right) .
$$

In the general case where both emitter and spectator are massive the correction term reads as

$$
\Delta I_{\gamma f, k}^{\text {coll }}\left(\mu_{j}, \mu_{k} ; \alpha\right)=-\frac{2(\alpha-1) c_{j} y_{+}+\left(c_{j}+2 y_{+}\right)\left(2 \alpha y_{+}+c_{j}\right)\left(\log \left(\frac{c_{j}+2 y_{+}}{2 \alpha y_{+}+c_{j}}\right)+4 \log (\alpha)\right)}{2\left(c_{j}+2 y_{+}\right)\left(2 \alpha y_{+}+c_{j}\right)}
$$

The limits $\mu_{j} \rightarrow 0$ and $\mu_{k} \rightarrow 0$ can be performed easily and one finds

$$
\Delta I_{\gamma f, k}^{\text {coll }}\left(\mu_{j}, 0 ; \alpha\right)=-\frac{1}{2}\left\{\frac{(\alpha-1)\left(\mu_{j}^{2}-1\right) \mu_{j}^{2}}{(\alpha-1) \mu_{j}^{2}-\alpha}-\log \left(-\alpha \mu_{j}^{2}+\alpha+\mu_{j}^{2}\right)+4 \log (\alpha)\right\},
$$

and

$$
\Delta I_{\gamma f, k}^{c o l l}\left(0, \mu_{k} ; \alpha\right)=-\frac{3}{2} \log (\alpha)
$$

respectively.

For these calculations we have used the HypExp package for Mathematica [71,72].

As mentioned above the $\alpha$-terms can as well be calculated using the formalism of mass regularization. To check our result we performed a second calculation using mass regularization and making use of the formulae giving in the appendix of [30] we found agreement between the two approaches.

\section{References}

[1] J. R. Andersen et al., "The SM and NLO multileg working group: Summary report," 1003.1241.

[2] T. Binoth et al., "A proposal for a standard interface between Monte Carlo tools and one-loop programs," Comput. Phys. Commun. 181 (2010) 1612-1622, 1001.1307. 
[3] S. Dittmaier, A. Kabelschacht, and T. Kasprzik, "Polarized QED splittings of massive fermions and dipole subtraction for non-collinear-safe observables," Nucl. Phys. B800 (2008) $146-189,0802.1405$.

[4] S. Höche, S. Schumann, and F. Siegert, "Hard photon production and matrix-element parton-shower merging," Phys. Rev. D81 (2010) 034026, 0912.3501.

[5] S. Dittmaier and M. Krämer, "Electroweak radiative corrections to $W$-boson production at hadron colliders," Phys. Rev. D65 (2002) 073007, hep-ph/0109062.

[6] C. M. Carloni Calame, G. Montagna, O. Nicrosini, and A. Vicini, "Precision electroweak calculation of the charged current Drell-Yan process," JHEP 12 (2006) 016, hep-ph/0609170.

[7] E. Accomando, A. Denner, and C. Meier, "Electroweak corrections to $W \gamma$ and $Z \gamma$ production at the LHC," Eur. Phys. J. C47 (2006) 125-146, hep-ph/0509234.

[8] M. Ciccolini, A. Denner, and S. Dittmaier, "Electroweak and QCD corrections to Higgs production via vector-boson fusion at the LHC," Phys. Rev. D77 (2008) 013002, 0710.4749.

[9] S. Actis, G. Passarino, C. Sturm, and S. Uccirati, "NLO Electroweak Corrections to Higgs Boson Production at Hadron Colliders," Phys. Lett. B670 (2008) 12-17, 0809.1301.

[10] A. Bredenstein, A. Denner, S. Dittmaier, and M. M. Weber, "Radiative corrections to the semileptonic and hadronic Higgs-boson decays $H \rightarrow W W / Z Z \rightarrow 4$ fermions," JHEP 02 (2007) 080, hep-ph/0611234.

[11] M. L. Ciccolini, S. Dittmaier, and M. Kramer, "Electroweak radiative corrections to associated W H and Z H production at hadron colliders," Phys. Rev. D68 (2003) 073003, hep-ph/0306234.

[12] G. Degrassi and F. Maltoni, "Two-loop electroweak corrections to Higgs production at hadron colliders," Phys. Lett. B600 (2004) 255-260, hep-ph/0407249.

[13] G. Degrassi and F. Maltoni, "Two-loop electroweak corrections to the Higgs-boson decay H $\rightarrow$ gamma gamma," Nucl. Phys. B724 (2005) 183-196, hep-ph/0504137.

[14] J. H. Kühn, A. Scharf, and P. Uwer, "Electroweak effects in top-quark pair production at hadron colliders," Eur. Phys. J. C51 (2007) 37-53, hep-ph/0610335.

[15] W. Bernreuther, M. Fücker, and Z.-G. Si, "Weak interaction corrections to hadronic top quark pair production: contributions from quark-gluon and $b \bar{b}$ induced reactions," Phys. Rev. D78 (2008) 017503, 0804.1237.

[16] M. Beccaria, G. Macorini, F. M. Renard, and C. Verzegnassi, "Associated t W production at LHC: A complete calculation of electroweak supersymmetric effects at one loop," Phys. Rev. D73 (2006) 093001, hep-ph/0601175.

[17] W. Hollik, M. Kollar, and M. K. Trenkel, "Hadronic production of top-squark pairs with electroweak NLO contributions," JHEP 02 (2008) 018, 0712.0287.

[18] W. Hollik, E. Mirabella, and M. K. Trenkel, "Electroweak contributions to squark-gluino production at the LHC," JHEP 02 (2009) 002, 0810.1044.

[19] J. Germer, W. Hollik, E. Mirabella, and M. K. Trenkel, "Hadronic production of squark-squark pairs: The electroweak contributions," JHEP 08 (2010) 023, 1004.2621.

[20] E. W. N. Glover and A. G. Morgan, "Measuring the photon fragmentation function at LEP," Z. Phys. C62 (1994) 311-322. 
[21] A. Denner, S. Dittmaier, T. Kasprzik, and A. Mück, "Electroweak corrections to $W+$ jet hadroproduction including leptonic W-boson decays," JHEP 08 (2009) 075, 0906.1656.

[22] S. Frixione, Z. Kunszt, and A. Signer, "Three-jet cross sections to next-to-leading order," Nucl. Phys. B467 (1996) 399-442, hep-ph/9512328.

[23] G. Somogyi, "Subtraction with hadronic initial states: an NNLO- compatible scheme," JHEP 05 (2009) 016, 0903.1218.

[24] S. Catani and M. H. Seymour, "A general algorithm for calculating jet cross sections in NLO QCD," Nucl. Phys. B485 (1997) 291-419, hep-ph/9605323.

[25] S. Catani, S. Dittmaier, M. H. Seymour, and Z. Trocsanyi, "The dipole formalism for next-to-leading order QCD calculations with massive partons," Nucl. Phys. B627 (2002) 189-265, hep-ph/0201036.

[26] D. A. Kosower, "Antenna factorization of gauge-theory amplitudes," Phys. Rev. D57 (1998) 5410-5416, hep-ph/9710213.

[27] J. M. Campbell, M. A. Cullen, and E. W. N. Glover, "Four jet event shapes in electron positron annihilation," Eur. Phys. J. C9 (1999) 245-265, hep-ph/9809429.

[28] A. Gehrmann-De Ridder, T. Gehrmann, and E. W. N. Glover, "Antenna subtraction at NNLO," JHEP 09 (2005) 056, hep-ph/0505111.

[29] A. Daleo, T. Gehrmann, and D. Maître, "Antenna subtraction with hadronic initial states," JHEP 04 (2007) 016, hep-ph/0612257.

[30] S. Dittmaier, "A general approach to photon radiation off fermions," Nucl. Phys. B565 (2000) 69-122, hep-ph/9904440.

[31] G. Ossola, C. G. Papadopoulos, and R. Pittau, "CutTools: a program implementing the OPP reduction method to compute one-loop amplitudes," JHEP 03 (2008) 042, 0711.3596.

[32] G. Ossola, C. G. Papadopoulos, and R. Pittau, "On the Rational Terms of the one-loop amplitudes," JHEP 05 (2008) 004, 0802.1876.

[33] C. F. Berger et al., "An Automated Implementation of On-Shell Methods for One- Loop Amplitudes," Phys. Rev. D78 (2008) 036003, 0803.4180.

[34] W. T. Giele and G. Zanderighi, "On the Numerical Evaluation of One-Loop Amplitudes: The Gluonic Case," JHEP 06 (2008) 038, 0805.2152.

[35] T. Binoth, J. P. Guillet, G. Heinrich, E. Pilon, and T. Reiter, "Golem95: a numerical program to calculate one-loop tensor integrals with up to six external legs," Comput. Phys. Commun. 180 (2009) 2317-2330, 0810.0992.

[36] P. Mastrolia, G. Ossola, T. Reiter, and F. Tramontano, "Scattering AMplitudes from Unitarity-based Reduction Algorithm at the Integrand-level," JHEP 08 (2010) 080, 1006.0710 .

[37] A. Denner and S. Dittmaier, "Reduction schemes for one-loop tensor integrals," Nucl. Phys. B734 (2006) 62-115, hep-ph/0509141.

[38] T. Stelzer and W. F. Long, "Automatic generation of tree level helicity amplitudes," Comput. Phys. Commun. 81 (1994) 357-371, hep-ph/9401258.

[39] F. Maltoni and T. Stelzer, "MadEvent: Automatic event generation with MadGraph," JHEP 02 (2003) 027, hep-ph/0208156. 
[40] J. Alwall et al., "MadGraph/MadEvent v4: The New Web Generation," JHEP 09 (2007) $028,0706.2334$.

[41] M. L. Mangano, M. Moretti, F. Piccinini, R. Pittau, and A. D. Polosa, "AlPGEN, a generator for hard multiparton processes in hadronic collisions," JHEP 07 (2003) 001, hep-ph/0206293.

[42] CompHeP Collaboration, E. Boos et al., "CompHEP 4.4: Automatic computations from Lagrangians to events," Nucl. Instrum. Meth. A534 (2004) 250-259, hep-ph/0403113.

[43] A. Pukhov, "CalcHEP 3.2: MSSM, structure functions, event generation, batchs, and generation of matrix elements for other packages," hep-ph/0412191.

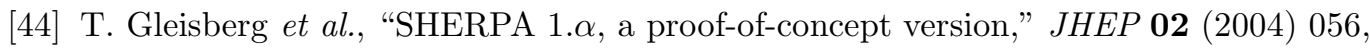
hep-ph/0311263.

[45] T. Gleisberg et al., "Event generation with SHERPA 1.1," JHEP 02 (2009) 007, 0811.4622.

[46] A. Cafarella, C. G. Papadopoulos, and M. Worek, "Helac-Phegas: a generator for all parton level processes," Comput. Phys. Commun. 180 (2009) 1941-1955, 0710.2427.

[47] W. Kilian, T. Ohl, and J. Reuter, "WHIZARD: Simulating Multi-Particle Processes at LHC and ILC," 0708.4233.

[48] T. Gleisberg and F. Krauss, "Automating dipole subtraction for QCD NLO calculations," Eur. Phys. J. C53 (2008) 501-523, 0709.2881.

[49] M. H. Seymour and C. Tevlin, "TeVJet: A general framework for the calculation of jet observables in NLO QCD," 0803.2231.

[50] M. Czakon, C. G. Papadopoulos, and M. Worek, "Polarizing the Dipoles," JHEP 08 (2009) $085,0905.0883$.

[51] K. Hasegawa, S. Moch, and P. Uwer, "AutoDipole: Automated generation of dipole subtraction terms," Comput. Phys. Commun. 181 (2010) 1802-1817, 0911.4371.

[52] K. Hasegawa, "Super AutoDipole," 1007.1585.

[53] R. Frederix, T. Gehrmann, and N. Greiner, "Automation of the Dipole Subtraction Method in MadGraph/MadEvent," JHEP 09 (2008) 122, 0808.2128.

[54] R. Frederix, S. Frixione, F. Maltoni, and T. Stelzer, "Automation of next-to-leading order computations in QCD: the FKS subtraction," JHEP 10 (2009) 003, 0908.4272.

[55] R. Frederix, T. Gehrmann, and N. Greiner, "Integrated dipoles with MadDipole in the MadGraph framework," JHEP 06 (2010) 086, 1004.2905.

[56] S. Dittmaier and M. Huber, "Radiative corrections to the neutral-current Drell-Yan process in the Standard Model and its minimal supersymmetric extension," JHEP 01 (2010) 060, 0911.2329 .

[57] K. P. O. Diener, S. Dittmaier, and W. Hollik, "Electroweak higher-order effects and theoretical uncertainties in deep-inelastic neutrino scattering," Phys. Rev. D72 (2005) 093002, hep-ph/0509084.

[58] A. D. Martin, R. G. Roberts, W. J. Stirling, and R. S. Thorne, "Parton distributions incorporating QED contributions," Eur. Phys. J. C39 (2005) 155-161, hep-ph/0411040. 
[59] K. Koller, T. F. Walsh, and P. M. Zerwas, "Testing QCD: direct photons in $e^{+} e^{-}$collisions," Zeit. Phys. C2 (1979) 197.

[60] S. Frixione, "Isolated photons in perturbative QCD," Phys. Lett. B429 (1998) 369-374, hep-ph/9801442.

[61] ALEPH Collaboration, D. Buskulic et al., "First measurement of the quark to photon fragmentation function," Z. Phys. C69 (1996) 365-378.

[62] A. Gehrmann-De Ridder, T. Gehrmann, and E. W. N. Glover, "Radiative Corrections to the Photon + 1 Jet Rate at LEP," Phys. Lett. B414 (1997) 354-361, hep-ph/9705305.

[63] A. Denner, S. Dittmaier, T. Gehrmann, and C. Kurz, "Electroweak corrections to hadronic event shapes and jet production in e+e- annihilation," Nucl. Phys. B836 (2010) 37-90, 1003.0986 .

[64] A. Denner, S. Dittmaier, M. Roth, and D. Wackeroth, "RACOONWW 1.3: A Monte Carlo program for four-fermion production at $e^{+} e^{-}$colliders," Comput. Phys. Commun. 153 (2003) 462-507, hep-ph/0209330.

[65] A. Denner, S. Dittmaier, M. Roth, and D. Wackeroth, "Predictions for all processes $e^{+} e^{-} \rightarrow$ 4 fermions + gamma," Nucl. Phys. B560 (1999) 33-65, hep-ph/9904472.

[66] A. Denner, S. Dittmaier, M. Roth, and D. Wackeroth, "Electroweak radiative corrections to $e^{+} e^{-} \rightarrow W W \rightarrow 4$ fermions in double-pole approximation: The RACOONWW approach," Nucl. Phys. B587 (2000) 67-117, hep-ph/0006307.

[67] A. Denner, S. Dittmaier, M. Roth, and M. M. Weber, "Electroweak radiative corrections to $e^{+} e^{-} \rightarrow t \bar{t} H, "$ Phys. Lett. B575 (2003) 290-299, hep-ph/0307193.

[68] A. Denner, S. Dittmaier, M. Roth, and M. M. Weber, "Radiative corrections to Higgs-boson production in association with top-quark pairs at $e^{+} e^{-}$colliders," Nucl. Phys. B680 (2004) 85-116, hep-ph/0309274.

[69] Z. Nagy and Z. Trocsanyi, "Next-to-leading order calculation of four-jet observables in electron positron annihilation," Phys. Rev. D59 (1999) 014020, hep-ph/9806317.

[70] Z. Nagy, "Next-to-leading order calculation of three-jet observables in hadron hadron collision," Phys. Rev. D68 (2003) 094002, hep-ph/0307268.

[71] T. Huber and D. Maitre, "HypExp 2, Expanding Hypergeometric Functions about HalfInteger Parameters," Comput. Phys. Commun. 178 (2008) 755-776, 0708.2443.

[72] T. Huber and D. Maitre, "HypExp, a Mathematica package for expanding hypergeometric functions around integer-valued parameters," Comput. Phys. Commun. 175 (2006) 122-144, hep-ph/0507094. 